Cette rubrique est dirigée par

Ph. MARGERIE

Ingénieur en chef du G.R.E.F.

Chargé de la snus-direction

"Bois-Papier-Imprimerie "

D. I. C. T. D.

Ministère de l'Industrie et de la Recherche

3, rue $B$ arbet-de-Jouy

75700 PARIS

\title{
LA PALETTE EN BOIS STRUCTURE DE PRODUCTION ET MARCHÉ
}

M. DUPREZ

Class. Oxford $83: 72$

La palette est un dispositif mobile, sur le plancher duquel peut être rassemblée une certaine quantité de marchandises pour constituer une unité de charge, en vue de sa manutention, de son transport et de son stockage à l'aide des appareils mécaniques de manutention tels que les transpalettes ou les chariots élévateurs à fourche.

Les bois utilisés couramment pour la construction des palettes sont des bois durs (chêne, hêtre, acacia...) qui résistent au choc et des bois tendres (pin maritime, sapin, peuplier...) d'un coût de production moins élevé. En fait la plupart des essences peuvent être utilisées, elles doivent cependant répondre à un certain nombre de caractéristiques : facilité de travail, densité, résistance aux attaques des champignons et des insectes...

En ce qui concerne les essences dures, la matière première utilisée pour la fabrication des palettes provient de la pointe des grumes qui correspond à la qualité $3^{e}$ choix. Cette qualité utilisée également pour la fabrication de bois sous rails et charpente provient pour $30 \%$ à $40 \%$ du traitement en taillis sous futaie et pour 15 à $20 \%$ du traitement en futaie.

En ce qui concerne les essences résineuses, la proportion de bois à palettes est plus importante, notamment en Aquitaine qui est la plus grosse région productrice de cette catégorie de bois.

La palette perdue, de construction sommaire est généralement réalisée avec les chutes de sciages tandis que la palette réutilisable est d'une conception plus soignée et d'une construction plus robuste.

Bien que la palette en bois tienne une place prépondérante dans la manutention industrielle des marchandises, elle subit la concurrence d’autres matériaux : métal, plastique, carton... Toutefois, le bois reste le mieux approprié technologiquem ant pour la fabrication des palettes.

\section{STRUCTURE DE LA PROFESSION DES FABRICANTS ET NÉGOCIANTS DE PALETTES EN BOIS}

Après avoir dressé l'inventaire des fabricants et négociants de palettes en bois, le Centre d'études de l'économie du bois (C.E.E.B.) a procédé à une enquête par voie postale auprès de ces professionnels. Les résultats de l'enquête sont les suivants : 
Les fabricants de palettes sont implantés sur tout le territoire mais la concentration la plus importante se localise en Rhône-Alpes, Aquitaine, Auvergne et Centre.

La production de palettes en bois n'est en général pas une activité exclusive dans l'entreprise, les entreprises spécialisées dans cette fabrication sont en minorité. L'activité principale de la branche " palette " est, dans la plupart des cas, la scierie.

L'activité palette au sein de l'entreprise emploie en moyenne $31 \%$ des effectifs et représente $33 \%$ du chiffre d'affaires.

La production de palettes et caisses palettes en bois s'élevait en 1974 à 19 millions d'unités utilisant environ $770000 \mathrm{~m}^{3}$ de bois sciés, $50 \%$ sont des palettes perdues, $43 \%$ sont des palettes réutilisables et $7 \%$ sont des caisses palettes. D'autre part, $70 \%$ des bois à palettes sont sciés dans l'entreprise, en conséquence, l'industrie de la fabrication de palettes est relativement intégrée.

La répartition du chiffre d'affaires et de la production par classes de production met en évidence l'importance numérique des petites et moyennes entreprises au sein de cette profession :

- $55 \%$ sont des petits fabricants (ayant produit moins de 50000 palettes en 1974) mais leur fabrication ne représente que $18 \%$ de la production globale;

- $41 \%$ de la production provient des entreprises de taille moyenne (50 000 à 150000 palettes) qui représentent $36 \%$ des fabricants.

- Les grosses entreprises (150000 à 700000 palettes) représentent en nombre $9 \%$ de la profession mais fabriquent $41 \%$ de la production globale.

\section{LE MARCHÉ FRANC̣AIS DE LA PALETTE EN BOIS}

Cette analyse a été effectuée à la suite d'une étude de marché réalisée par le Centre d'études de l'économie du bois auprès d'un échantillon diversifié de fabricants, négociants et utilisateurs de palettes en bois.

Il a été procédé à une cinquantaine d'interviews sur la base d'entretiens " non directifs » qui comportent des questions "ouvertes " en majeure partie, celles-ci permettant à la personne interrogée de s'exprimer librement sur un certain nombre de thèmes.

L'industrie de la palette joue un rôle essentiel dans la rationalisation des systèmes de distribution des produits. Par ailleurs, elle s'approvisionne en bois de qualité secondaire qui n'ont guère d'autres utilisations.

Parmi les principaux secteurs utilisateurs de palettes, on peut citer : les matériaux de construction, le bâtiment, la chimie, la verrerie, la métallurgie, les fabricants de métaux, l'imprimerie, la papeterie, l'industrie alimentaire...

Le marché de la palette en bois a connu un boom entre 1965 et 1974 dû à la palettisation des grosses et moyennes entreprises.

Cette forte demande a favorisé la multiplication des fabricants de palettes, en particulier des scieurs se sont lancés dans cette production.

II semble cependant que les prochaines années ne connaîtront plus qu'un accroissement modéré de la demande de palettes correspondant à l'équipement des petites entreprises et au renouvellement des secteurs déjà palettisés.

Dans la conjoncture actuelle, les utilisateurs seront beaucoup plus soucieux de la qualité du produit, surtout avec le développement des systèmes automatisés de manutention et de stockage.

La standardisation va sans doute progresser; une norme semble devoir s'affirmer. II s'agit de la $1000 \times 1200$, car ses dimensions correspondent aux nécessités d'un grand nombre de secteurs.

Parallèlement on assistera à l'extension des pools et à la mise sur pied de nouveaux procédés de gestion des palettes (par exemple location, vente-rachat...). Standardisation et pools sont deux éléments favorables au développement de la palette de réemploi. 
La palette recyclable, compromis entre la palette perdue et la palette récupérable, devrait connaitre une progression certaine. Mais la palette perdue maintiendra sa production au moins à son volume actuel alors que sa part relative sur le marché diminuera.

La concurrence d'autres matériaux que le bois pour la fabrication des palettes (plastique, métal...) ne devrait pas être trop vive et leur part sur le marché ne devrait s'accroître que lentement.

Le déclin de la palette et son remplacement par d'autres procédés de manutention pourrait intervenir dans une quinzaine d'années.

L'industrie de la palette en bois atteindra à court terme sa phase de maturité et les capacités de production ne devraient être accrues qu'avec discernement.

Il est opportun de rappeler qu'il est souhaitable pour une entreprise d'avoir plusieurs produits situés à différentes phases de leur cycle de vie.

\section{LE MARCHÉ INTERNATIONAL DE LA PALETTE}

Les informations sur le marché international de la palette proviennent pour une large part du Congrès mondial de la palette qui s'est tenu à Montreux, en février 1975.

La production mondiale de palettes en 1974 serait de l'ordre de 350 millions d'unités. Les principaux pays producteurs seraient : les U.S.A. (204 millions), le Japon (30 millions), la France (20 millions), l'Allemagne (19 millions), la Grande-Bretagne ( 17,5 millions), le Canada (10 millions).

Les spécialistes estiment que les pays très industrialisés ont atteint un très haut degré de palettisation voire même une palettisation absolue. Le taux de croissance ne dépassera guère $10 \%$ dans l'avenir à condition que la production industrielle ou le volume des exportations ne stagnent pas trop.

Par contre, dans des pays faiblement palettisés comme l'Australie, le Japon, le Canada, on escompte un taux de croissance très soutenu. C'est ainsi que les Japonais évaluent ce taux à $39 \%$ par an pour la prochaine période.

La plupart des pays producteurs de palettes ne connaissent pas de réelles difficultés dans l'approvisionnement en bois. Notons toutefois quelques exceptions comme la Grande-Bretagne, le Japon qui sont très dépendants des importations.

Dans de nombreux pays, des recherches sont entreprises pour trouver de nouvelles méthodes d'exploitation du bois et d'intensification des rendements.

A l'étranger et parmi les principaux pays producteurs de palettes, les systèmes de distribution sont en général plus sophistiqués qu'en France. Ainsi en Australie, au Japon, en R.F.A. et aux U.S.A., les pools privés sont plus répandus et fonctionnent souvent sur la base de location de palettes.

Si des progrès ont été accomplis dans l'établissement des normes, il semble que l'on soit encore très éloigné de la normalisation mondiale. Cependant les différents pays s'emploient à réduire le nombre de formats des palettes et l'extension des pools est un élément moteur dans ce domaine.

Michèle DUPREZ

CENTRE D'ÉTUDES ET DE L'ÉCONOMIE DU BOIS

1. place du Théâtre-Français

75001 PARIS 